Open Access

\title{
A vascular endothelial growth factor receptor gene variant is associated with susceptibility to acute respiratory distress syndrome
}

Natalia Hernandez-Pacheco ${ }^{1 \dagger}$, Beatriz Guillen-Guio ${ }^{1 \dagger}$, Marialbert Acosta-Herrera ${ }^{1,2,13}$, Maria Pino-Yanes ${ }^{1,3,4}$, Almudena Corrales ${ }^{1,3}$, Alfonso Ambrós ${ }^{5}$, Leonor Nogales ${ }^{6}$, Arturo Muriel', Elena González-Higueras ${ }^{8}$, Francisco J. Diaz-Dominguez ${ }^{9}$, Elizabeth Zavala ${ }^{3,10}$, Javier Belda ${ }^{11}$, Shwu-Fan Ma ${ }^{12}$, Jesús Villar ${ }^{2,3}$, Carlos Flores ${ }^{1,3^{*}}$ and the GEN-SEP Network

\footnotetext{
* Correspondence: cflores@ull.edu.es ${ }^{\dagger}$ Natalia Hernandez-Pacheco and Beatriz Guillen-Guio contributed equally to this work.

'Research Unit, Hospital

Universitario N.S. de Candelaria, Universidad de La Laguna, Santa Cruz de Tenerife, Spain

${ }^{3}$ CIBER de Enfermedades Respiratorias, Instituto de Salud Carlos III, Madrid, Spain

Full list of author information is available at the end of the article
}

\begin{abstract}
Background: The acute respiratory distress syndrome (ARDS) is one of the main causes of mortality in adults admitted to intensive care units. Previous studies have demonstrated the existence of genetic variants involved in the susceptibility and outcomes of this syndrome. We aimed to identify novel genes implicated in sepsisinduced ARDS susceptibility.

Methods: We first performed a prioritization of candidate genes by integrating our own genomic data from a transcriptomic study in an animal model of ARDS and from the only published genome-wide association study of ARDS study in humans. Then, we selected single nucleotide polymorphisms (SNPs) from prioritized genes to conduct a case-control discovery association study in patients with sepsis-induced ARDS $(n=225)$ and population-based controls $(n=899)$. Finally, we validated our findings in an independent sample of 661 sepsis-induced ARDS cases and 234 at-risk controls.

Results: Three candidate genes were prioritized: dynein cytoplasmic-2 heavy chain-1, fms-related tyrosine kinase 1 (FLT1), and integrin alpha-1. Of those, a SNP from FLT1 gene (rs9513106) was associated with ARDS in the discovery study, with an odds ratio (OR) for the $C$ allele of $0.76,95 \%$ confidence interval (Cl) $0.58-0.98(p=0.037)$. This result was replicated in an independent study $(\mathrm{OR}=0.78,95 \% \mathrm{Cl}=0.62-0.98, p=0.039)$, showing consistent direction of effects in a meta-analysis ( $\mathrm{OR}=0.77,95 \% \mathrm{Cl}=0.65-0.92, p=0.003)$.

Conclusions: We identified FLT1 as a novel ARDS susceptibility gene and demonstrated that integration of genomic data can be a valid procedure to identify novel susceptibility genes. These results contribute to previous firm associations and functional evidences implicating FLT1 gene in other complex traits that are mechanistically linked, through the key role of endothelium, to the pathophysiology of ARDS.
\end{abstract}

Keywords: Acute lung injury, Sepsis, Polymorphism, Genetic predisposition 


\section{Background}

The acute respiratory distress syndrome (ARDS) is an acute and intense inflammatory process of the lung caused by injury to the alveolar-capillary membrane that results in increased permeability and protein-rich edema. Diagnosis is based on a constellation of clinical, radiographic, and physiologic abnormalities including severe hypoxemia, bilateral pulmonary infiltrates on chest-X-ray, and no clinical evidence of hydrostatic/cardiogenic pulmonary edema [1]. The estimated annual incidence of ARDS is 7 cases per 100,000 individuals, with an associated hospital mortality of 40\% [2, 3]. Although there are different processes that can cause ARDS, such as pneumonia, severe trauma, or blood transfusions [4]; sepsis continues to be the main risk factor precipitating this syndrome in adults [5].

Several studies have demonstrated the influence of the individual's genetic variation in the susceptibility to ARDS [6,7]. It is suggested that such genetic factors not only could partially account for the heterogeneity of ARDS presentation and the variability in the response to therapies among patients, but also may explain the differential prevalence of ARDS among different populations [4, 8] and ethnic groups [2, 9-11]. A number of genes have been proposed as biological candidates for ARDS susceptibility based on gene expression studies in animal or cellular models followed by candidate gene association studies [6].

Despite the fruitful use of genome-wide association studies (GWAS) to unravel risk factors in many complex diseases, to date only one GWAS in patients with ARDS has been published and it was focused on trauma-induced ARDS. In that study, the protein tyrosine phosphatase, receptor type F-interacting protein alpha-1 (PPFIA1) gene was identified as a novel ARDS locus, which encodes a protein implicated in cell-cell junctions and cell interactions with the extracellular matrix [12]. The authors first identified the most significant association signals in the discovery stage, and then, a replication was performed in independent samples. Finally, these signals were recognized by integration with gene expression data [12]. However, it is anticipated that many other ARDS loci remain to be identified. In the present study, we aimed to reassess the GWAS results published by Christie et al. [12] performing the integration with transcriptomic data from an animal model of ARDS. This approach allowed to prioritize candidate genes that were followed-up in independent replication studies in order to reveal novel sepsis-induced ARDS susceptibility loci.

\section{Methods}

\section{Prioritization of candidate genes}

Figure 1 illustrates the overall scheme of the process involved in the selection of candidate genes. Gene prioritization began with transcriptomic data derived from an experimental animal model of ARDS, which was induced by abdominal sepsis after $24 \mathrm{~h}$ of cecal ligation and puncture, and subsequent challenge by mechanical ventilation (MV) strategies for $4 \mathrm{~h}$ [13] (ArrayExpress accession E-MTAB-2673). Three experimental groups were compared based on the ventilatory approach applied: unventilated spontaneously breathing septic animals (SS), septic animals ventilated with protective MV (SPV), and septic animals ventilated with injurious MV (SIV). Total RNA was purified from total left lung tissue of surviving animals from each experimental group, which 


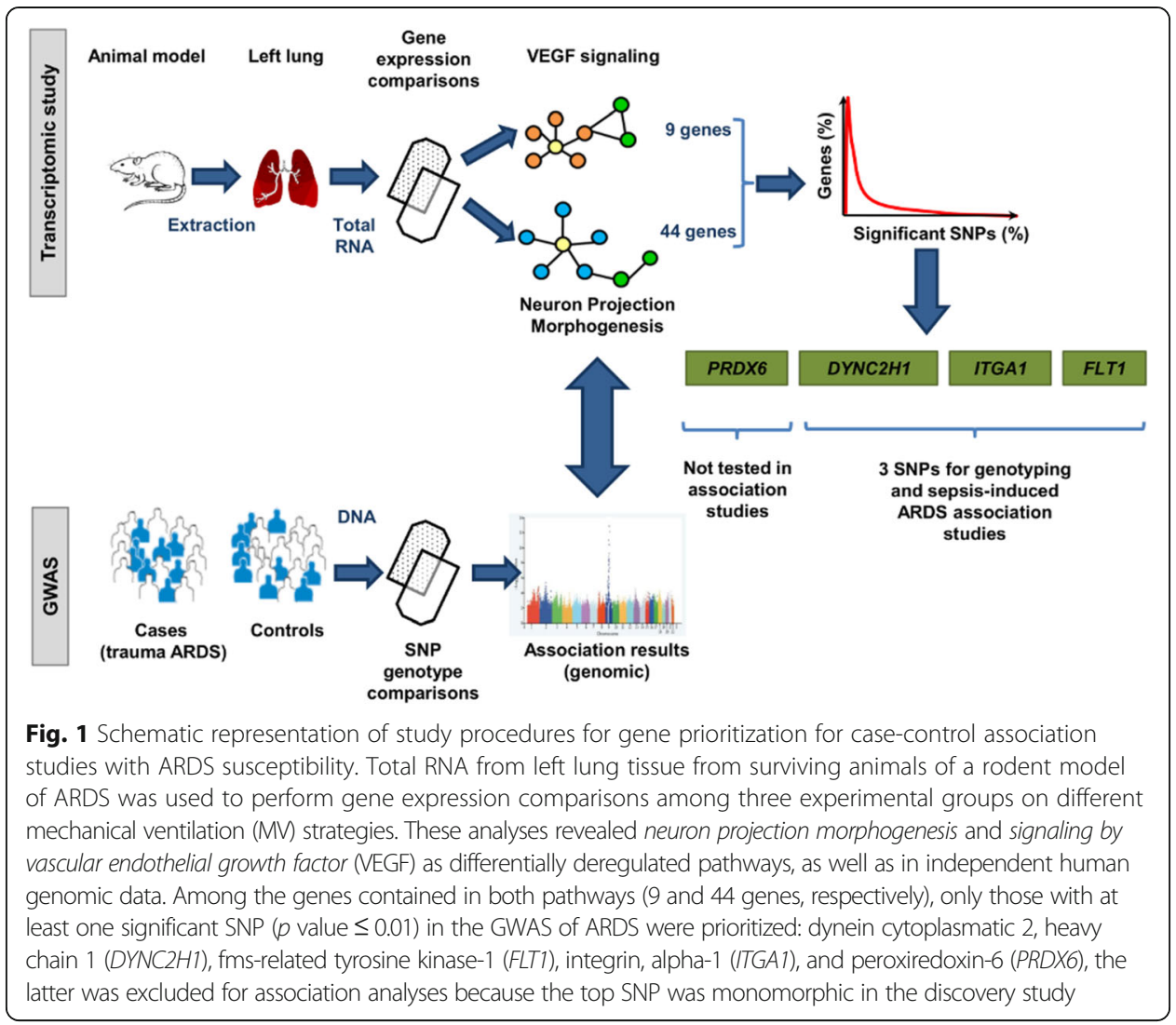

was used for hybridization of fragmented cRNA to the GeneChip Rat Genome 2302.0 Array (Affymetrix, Santa Clara, CA). Then, differential gene expression was identified using a multi-group comparison by means of MulCom test, revealing 2859 deregulated genes with a false discovery rate $(F D R) \leq 0.05$ and fold change $(F C) \geq 1.7$ (Additional file 1: Table S1). Differential contribution on biological processes of these genes was assessed by functional annotation clustering analyses using the Database for Annotation, Visualization and Integrated Discovery (DAVID) v6.7 [14]. This analysis revealed Neuron projection morphogenesis as the key deregulated pathway $\left(\mathrm{FDR}=1.76 \times 10^{-3}\right)$ (Additional file 2: Table S2). Later, in order to reveal other common mechanisms in the animal model, we performed a protein-protein interaction network with the EnrichNet tool using the overlapping deregulated genes from the multi-group comparison (Additional file 3: Table S3). While eight signaling interaction networks were significant in at least one of the groups, the signaling by vascular endothelial growth factor (VEGF) network was the only significantly deregulated across all three groups. Signaling by VEGF plays a central and broad role in endothelial cell function, and in several aspects of the immune and inflammatory response, usually linked to lung edema promotion [15]. Moreover, neuron projection morphogenesis shares important characteristics with signaling by VEGF, such as the interplay of similar angiogenic molecules, principles, and mechanisms implicated in the navigation through their targets and morphogens [16]. In fact, VEGF could be considered a prototypic angioneurin, comparable to semaphorins, netrins, slits, ephrins, and neuropilins [17]. 
To validate these results, we then performed a post hoc evaluation from two independent public genomic studies of ARDS. The first was a transcriptomic study in blood cells from septic and sepsis-derived ARDS patients that had received MV for $48 \mathrm{~h}$ or less [18] (NCBI accession number GSE10474), which resembled the most experimental conditions from our animal model at that time (Additional file 4: Table S4). The second was the only genome-wide association study (GWAS) of ARDS published at the time [12] (https://doi.org/10.1371/journal.pone.0028268.s003). In these two datasets, we observed that genes involved in neural and vascular functions were deregulated and nominally associated with ARDS.

The two pathways, neuron projection morphogenesis (Gene Ontology identifier GO:0048812) [19] and signaling by VEGF (KEGG identifier map04370) [20], contained 9 and 44 genes, respectively. These were followed up to prioritize the candidate genes for association studies. We then surveyed all SNPs in these 53 genes (including $100 \mathrm{ki}$ lobases $(\mathrm{Kb})$ flanking sequences [21]) reported by Christie et al. [12] and retained the ones showing a $p$ value $\leq 0.01$. To take into account the multiple testing and to avoid potential prioritization bias due to gene length, we corrected the significance by the independent number of variants analyzed per gene, which was calculated with the SNPSpD software [22] based on the linkage disequilibrium (LD) information in the European population data from The 1000 Genomes Project [23].

Finally, either the most significant SNP or a SNP in strong LD among those genes surviving multiple testing corrections was put forward for association studies. LD patterns were obtained with Haploview in the form of pairwise $r^{2}$ values [24].

\section{Association study: discovery}

A case-control study was performed in DNA samples from unrelated Spanish individuals. As cases, we studied 429 patients with severe sepsis admitted into a network of intensive care units (ICUs) in Spain (see "Acknowledgements") who were followed for the development of ARDS ( $n=230)$, according to the definition of the American-European Consensus Criteria [1] and as having moderate or severe ARDS according to the Berlin criteria [25]. All patients were managed with a lung-protective MV strategy using low tidal volume (4-8 $\mathrm{ml} / \mathrm{kg}$ predicted body weight) and PEEP $\geq 5 \mathrm{cmH}_{2} \mathrm{O}$ at the time of meeting ARDS definition. Controls were derived from a population-based study with nationwide recruitment by the Spanish National DNA Biobank [26] and consisted of 900 individuals self-reporting of having four grandparents of Spanish origin and with no history of respiratory disease or major infections. DNA was extracted using the GFX kit (GE Healthcare, Little Chalfont, UK) following the manufacturer's recommendations. Genotyping of the SNPs selected was performed with KASP ${ }^{\mathrm{m} w}$ probes (LGC Genomics, Teddington, UK) using a 7500 Fast Real-Time PCR System (Life Technologies, Carlsbad, CA). Genotype calls were automatically assigned by discriminating analysis with $95 \%$ confidence using the SDS software (Life Technologies). A total of 60 samples were genotyped by duplicate to monitor the genotyping reproducibility by measuring the concordance rate. SNP genotyping quality control and deviations from the Hardy-Weinberg equilibrium (HWE) were assessed with R 3.2.2 [27]. SNP minor allele frequency (MAF) in the control group was calculated and compared to the values reported for the Iberian Population in Spain from The 1000 Genomes Project to assess its consistency [23]. 
We performed association analyses of the individual SNPs with ARDS susceptibility using logistic regressions models in $R$, comparing ARDS cases and population-based controls that were retained after quality controls (Fig. 2 and Table 1).

\section{Association study: replication}

Replication was assessed in an independent sample set obtained from the database of Genotypes and Phenotypes (dbGaP) from a GWAS by the ARDSnet and the iSPAAR Consortium (study accession phs000631.v1.p1). According to the information deposited in the dbGaP database, a total of 518,313 SNPs were genotyped using the Illumina genotyping platform Human660W-Quad v1.0 in 2235 Western European descent individuals, including 1122 ARDS cases and 1113 at-risk controls (i.e., patients with pneumonia, trauma, and pancreatitis or those subjected to aspiration or massive blood transfusion but did not develop ARDS). We performed quality control procedures with PLINK v1.07 [28] to remove samples with missing clinical information and genotype call rates < 95\%, leaving a total of 1119 cases and 1113 controls for further analyses. Additionally, those SNPs with a genotyping rate $<97 \%$ were excluded, leaving a total of 516,348 SNPs, none of which deviated from HWE $\left(p\right.$ value $\left.<1 \times 10^{-6}\right)$. In addition, principal component analysis was performed with EIGENSOFT (v4.2) [29] analyzing $\sim 100,000$ independent SNPs extracted from this data. In order to retrieve the SNPs of interest, genotype imputation was performed with Minimac3 [30] using the Haplotype Reference Consortium version r1.1 data as reference panel [31] on the Michigan Imputation Server [32]. Association testing was conducted using logistic regression models with $\mathrm{R}$ including the first two principal components as covariates, showing a minimal inflation due to population stratification (Lambda =1.036). To approximate as much as possible this study to that utilized in the discovery stage, the replication only considered as cases those patients with sepsis-associated ARDS $(n=661)$ and as controls those patients that were free from sepsis and ARDS $(n=234)$ (Fig. 2). Detailed demographic and clinical data from the subset of patients included in this stage can be found in Table 2.

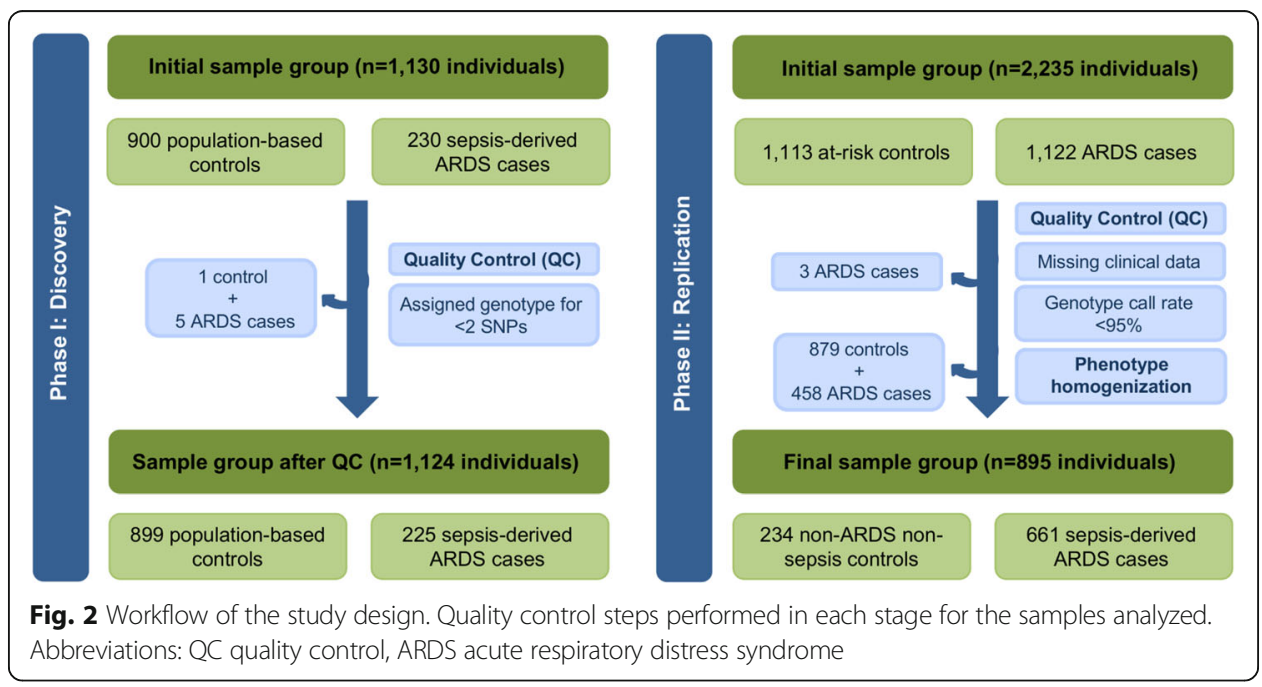


Table 1 Characteristics of ARDS patients and controls of the discovery study

\begin{tabular}{|c|c|c|}
\hline Characteristics & Cases $(n=225)$ & Controls $(n=889)$ \\
\hline Gender (\% male) & 37 & 41 \\
\hline Mean age (years) $\left(P_{25}-P_{75}\right)$ & $63(54-75)$ & $41(32-49)$ \\
\hline Hypertension (\%) & 44 & 3 \\
\hline Smoker (\%) & 30 & 49 \\
\hline Previous surgery (\%) & 53 & NA \\
\hline Ischemic cardiac disease (\%) & 11 & NA \\
\hline \multicolumn{3}{|l|}{ Source of sepsis (\%) } \\
\hline Pulmonary & 49 & NA \\
\hline Extrapulmonary & 51 & NA \\
\hline \multicolumn{3}{|l|}{ Pathogen (\%) } \\
\hline Gram-negative & 49 & NA \\
\hline Gram-positive & 33 & NA \\
\hline Gram-negative and Gram-positive & 5 & NA \\
\hline Polymicrobial & 2 & NA \\
\hline Virus & 6 & NA \\
\hline Fungi & 3 & NA \\
\hline \multicolumn{3}{|l|}{ Organ dysfunction (\%) } \\
\hline Circulatory & 62 & NA \\
\hline Renal & 42 & NA \\
\hline Hepatic & 18 & NA \\
\hline Neurologic & 22 & NA \\
\hline Coagulation & 22 & NA \\
\hline APACHE $\|$ (median) $\left(P_{25}-P_{75}\right)$ & $22(18-27)$ & NA \\
\hline ICU mortality (\%) & 44 & NA \\
\hline
\end{tabular}

APACHE II Acute Physiology and Chronic Health Evaluation II, ARDS acute respiratory distress syndrome, ICU intensive care unit, $P_{25}$ percentile $25, P_{75}$ percentile $75, N A$ not available

\section{Meta-analysis of associations and evaluation of functionality}

A meta-analysis was performed with the METASOFT software [33] to estimate the overall effect size of associated SNPs across the two studies (discovery and replication cohorts) and to evidence the existence of heterogeneity of effects using Cochran's $Q$ statistic $p$ value. Statistical power to detect effects larger that an odds ratio (OR) of 1.35 for a $\mathrm{MAF}=22 \%$ at 0.01 significance threshold was estimated as $93.3 \%$ (http:// osse.bii.a-star.edu.sg/index.php).

To assess the functional role of the SNPs, the online tool Haploreg v4.1 [34] was used to query the empirical data provided by the Encyclopedia of DNA Elements (ENCODE) project. Moreover, updated information related to expression Quantitative Trait Loci (eQTL) were inspected for the SNPs selected for each gene included in the association analyses or any SNP in large LD $\left(r^{2} \geq 0.8\right)$. For that, several publicly available datasets were explored, including the GTEx Portal [35] and SiNoPsis v1.0 (https://compgen.bio.ub.edu/ SiNoPsis/).

\section{Results and discussion}

Thirteen out of 53 genes from the signaling by VEGF and neuron projection morphogenesis pathways showed at least one SNP associated with trauma-induced ARDS in the 
Table 2 Characteristics of ARDS patients and controls of the replication study

\begin{tabular}{|c|c|c|c|c|}
\hline \multirow[t]{2}{*}{ Characteristics } & \multicolumn{2}{|c|}{ Complete dataset $(n=2235)^{a}$} & \multicolumn{2}{|c|}{ Analyzed dataset $(n=895)^{\mathrm{b}}$} \\
\hline & $\begin{array}{l}\text { Cases } \\
(n=1122)\end{array}$ & $\begin{array}{l}\text { Controls } \\
(n=1113)\end{array}$ & $\begin{array}{l}\text { Cases } \\
(n=661)\end{array}$ & $\begin{array}{l}\text { Controls } \\
(n=234)\end{array}$ \\
\hline Gender (\% male) & 56 & 61 & 58 & 67 \\
\hline Mean age (years) $\left(P_{25}-P_{75}\right)$ & $56(44-70)$ & $62(51-75)$ & $60(48-73)$ & $58(45-73)$ \\
\hline Ventilator-free days (median) $\left(P_{25}-P_{75}\right)$ & $17(0-24)$ & $27(24-29)$ & $14(0-23)$ & $27(24-28)$ \\
\hline Mortality (\%) & 23 & 6 & 28 & 5 \\
\hline
\end{tabular}

GWAS data at the nominal significance level reported in the published study $(p \leq 0.01)$ (Fig. 1; Additional file 5: Table S5). After correcting for the independent number of SNPs analyzed in each gene, four genes contained the largest proportion of significant SNPs normalized by number of variants analyzed per gene, namely dynein cytoplasmatic 2, heavy chain 1 (DYNC2H1), fms-related tyrosine kinase-1 (FLT1), integrin, alpha-1 (ITGA1), and peroxiredoxin-6 (PRDX6). For each of them, the most significant SNP, or a SNP in high LD with it, was chosen for genotyping. For DYNC2H1 and ITGA1, we selected the SNPs $\operatorname{rs} 11225640\left(p=3.5 \times 10^{-5}\right.$ with trauma-induced ARDS) and rs16880534 ( $p=4.7 \times 10^{-3}$ with trauma-induced ARDS), respectively. However, for the FLT1 gene, the flanking region of the top-prioritized SNP (rs9508026, $p=1.1 \times 10^{-3}$ with trauma-induced ARDS) did not allow the design of a genotyping assay. Therefore, this top-associated SNP was captured by an alternative SNP (rs9513106) in strong LD $\left(r^{2}=0.8\right)$. Lastly, for PRDX6, we selected the variant rs9425722 $\left(p=3.2 \times 10^{-3}\right.$ with trauma-induced ARDS).

In the discovery study, association testing was performed in 1124 individuals (225 sepsis-induced ARDS cases and 899 controls) after removing six subjects (1 control and 5 cases) whose genotype could not be determined for $\geq 2$ SNPs (Fig. 2). Among cases, $49 \%$ had a positive blood culture, where Gram-negative bacteria were the causing agents of sepsis. Additionally, the most likely origin of sepsis was pulmonary (49\% of patients), and the overall ICU mortality rate was $44 \%$ (Table 1 ).

The PRDX6 variant selected (rs9425722) resulted monomorphic in our samples (results not shown). This result was consistent with the information found in the European population data from The 1000 Genomes Project [23], where this position is monomorphic. For this reason, and given that a previous study evaluated the common variants from the PRDX6 gene and found no association with ARDS [36], we disregarded this gene from further analyses. The other three SNPs selected for association studies had a genotype concordance rate $>98 \%$ among duplicated samples and a genotyping rate $>95 \%$ (Table 3 ), and none of the SNPs deviated significantly from the HWE in the population-based controls. Association testing of these three SNPs with the development of ARDS susceptibility (Additional file 6: Table 4) revealed a significant association for the FLT1 SNP (rs9513106), with an OR for the C allele of 0.76 (95\% confidence interval $(\mathrm{CI})=0.58-0.98, p=0.037$ ). This protective effect of the $\mathrm{C}$ allele was validated in the replication study (661 sepsis-associated ARDS cases and 234 controls) showing an OR for the $\mathrm{C}$ allele of $0.78(95 \% \mathrm{CI}=0.62-0.98)$ and a $p=0.039$. No heterogeneity of effects was found between the two studies (Cochran's $Q, p=0.850$ ) and 
Table 3 Summary metrics and allelic frequencies of genotyped SNPs in the discovery study

\begin{tabular}{|c|c|c|c|c|c|c|c|}
\hline \multirow[t]{2}{*}{ SNP } & \multirow[t]{2}{*}{ Gene } & \multirow{2}{*}{$\begin{array}{l}\text { Minor } \\
\text { allele }\end{array}$} & \multirow{2}{*}{$\begin{array}{l}\text { Concordance } \\
\text { rate }(95 \% \mathrm{Cl})\end{array}$} & \multirow{2}{*}{$\begin{array}{l}\text { Completion } \\
\text { rate }(\%)\end{array}$} & \multirow[t]{2}{*}{ HWE ${ }^{a}$} & \multicolumn{2}{|l|}{$\mathrm{MAF}^{\mathrm{b}}$} \\
\hline & & & & & & Cases & Controls \\
\hline rs9513106 & FLT1 & C & $100(95.7-100)$ & 95.0 & 0.213 & 0.221 & 0.270 \\
\hline rs11225640 & DYNC2H1 & C & 98.9 (94.1-99.8) & 97.9 & 0.428 & 0.103 & 0.116 \\
\hline rs16880534 & ITGA1 & G & $100(95.9-100)$ & 99.6 & 0.117 & 0.216 & 0.199 \\
\hline
\end{tabular}

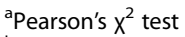

${ }^{\mathrm{b}}$ Minor allele frequency

concordance in the direction of effects was confirmed in a fixed-effect meta-analysis comprising a total of 886 sepsis-induced ARDS cases and 1133 controls $(O R=0.77,95 \%$ $\mathrm{CI}=0.65-0.92, p=0.003)$.

The FLT1 gene encodes a tyrosine kinase receptor with an extracellular ligand-binding region with seven domains of immunoglobulin that belongs to the VEGF receptor family. Ligands of FLT1, such as VEGF-A, VEGF-B, and placental growth factors are critically involved in edema formation during ARDS [15]. After binding FLT1, they trigger intracellular signaling pathways involved in cellular proliferation, survival, and migration, as well as in the increase of endothelial cell permeability and angiogenesis [37]. Studies carried out in animal models support the implication of this pathway promoting vascular permeability [38], and functional assays in human vascular cells have shown that elevated expression of FLT1 gene was associated with decreased amounts of inflammatory adhesion molecules [39]. VEGF signaling is required for the maintenance of adult lung alveolar structures and reduced VEGF levels have been found in bronchoalveolar lavage (BAL) samples from ARDS patients [40-42], in contrast with the high VEGF levels found in BAL from healthy individuals [43]. Furthermore, oxidative stress, cytokines, and hypoxia are important factors that regulate VEGF expression [44], which are key players in ARDS development. Therefore, a positive feedback may exist between all or some of these factors to increase VEGF expression, and consequently, VEGF binding to its receptors may contribute to the promotion of increased vascular permeability and a higher degree of hypoxemia as it has been reported in the earliest stages of ARDS [45]. Common variants in VEGF signaling genes have been consistently associated with susceptibility or outcomes of ARDS patients, and VEGF is one of the most replicated genes associated with ARDS [6]. However, to date, variants from FLT1 have never been associated with ARDS susceptibility or outcomes, although some of them have been found to be associated with other relevant complex traits. In this sense, the FLT1 variant identified in this study was previously associated in two GWAS of large sample sizes and with multi-ethnic patients with coronary arterial disease [46, 47], where endothelium also plays a key pathophysiological role. Besides, a recent study has shown that two FLT1 variants are associated with a reduced count of red blood cells [48], and with risk of preeclampsia, perhaps influencing the maternal endothelial dysfunction $[48,49]$. Taken together, this information supports the firm role of FLT1 gene variants in complex traits that are mechanistically linked, through the key role of endothelium, to the pathophysiology of the ARDS.

Our findings reinforce the critical importance of variants in genes from the VEGF pathway in ARDS susceptibility. Despite the fact that the FLT1 SNP rs9513106 is located in an intronic region, the nucleotide change predicts alterations in two transcription regulatory motifs for the members binding to the GATA DNA elements and signal 
transducer and activator of transcription (STAT) factor families according to Haploreg v4.1. However, no significant eQTL results were found for rs9513106 or any SNP in strong LD in Europeans. In addition, as demonstrated by experiments performed as part of the ENCODE project, rs9513106 is in strong LD with another intronic SNP 9.6 Kb upstream, rs9508025. This SNP is located in a highly conserved region among species, in particular, in an enhancer histone mark and a DNAse I hypersensitivity site in fetal lung cells. Interestingly, rs9508025 was identified as one of the top-hits associated with coronary artery disease risk in a large GWAS study [50]. Nevertheless, future studies are needed to assess the functional effects of these variants in FLT1 expression, protein activity, or its relation with the soluble forms of the protein (i.e., sFLT1) in patient samples. It should be mentioned that increased levels of sFLT1 in plasma have been related with sepsis severity, organ dysfunction, and mortality [51, 52]. Additionally, high sFLT1 levels have also been found in BAL samples of ARDS patients [53]. Therefore, given that sFLT1 acts as a natural competitive inhibitor of VEGF [54], we postulate that the protective genetic variant found in FLT1 (or a variant in LD with it) could promote the increase of sFLT1 levels and hence, contributing to the protection from lung injury by reducing VEGF activity and the vascular permeability in ARDS patients. Further studies will be needed to evaluate this possibility.

The main strength of our study is that we found an association of the FLT1 SNP rs9513106 with sepsis progression towards ARDS in the discovery sample using population-based controls and that this SNP was consistently replicated in an independent sample using hospitalized at-risk controls. Therefore, the effect of environmental factors related with the hospital setting or study design on the association of the FLT1 variant with ARDS susceptibility is minimal. In addition, since no adjustment for population stratification was performed in the discovery sample, the replication in an independent sample with appropriate adjustments suggests minimal confounding effects due to population stratification. However, the top prioritized finding in the published GWAS of ARDS (rs9508026) [12] was not tested due to the fact that this did not allow the design of a genotyping assay so that, an alternative SNP was captured. In addition, although we prioritized the top significant SNP, we only considered one variant per gene for association studies. Therefore, other genetic variants not analyzed in our study (including rarer variants) could also be relevant in this syndrome, and this limitation could explain the lack of significance in the results for the other three genes.

Regarding the control dataset used in the discovery phase, population-based controls are considered the most obvious control group, being a random sample from the source population where cases were obtained [55]. The use of this kind of controls allows reducing the possible selection bias caused by differences among cases and controls beyond the disease process itself and potential risk factors [56, 57]. Additionally, they provide stronger association effect estimates than hospital-based controls when cases have been recruited from hospitals since the association evidence revealed by population-based controls include those obtained by using controls from the hospital [56]. As a drawback, potential confounders related to the differences in age and comorbidities among cases and the population-based controls may have affected the findings of the discovery study. However, the results replicated in the independent ARDSnet/ iSPAAR Consortium study, where no differences in age and comorbidities were 
detected (controls were patients at-risk) allowing to control more closely the environmental factors. This strongly reinforces the validity of our findings.

We propose that the prioritization of genes based on pathway overlapping among distinct data sources (animal and human) and studies (transcriptomics and GWAS) would provide firm deregulated mechanisms during the acute lung injury, leading to the selection of more robust genes, contrasting to classical association studies that focus on a biological candidate based on literature information. For now, our studies preclude concluding whether or not the FLT1 gene variant is directly associated with sepsis-induced ARDS susceptibility or with the aggravation of sepsis. However, whatever the case, it is equally valid and relevant information. Further studies would be needed to disentangle the main driver of such association.

\section{Conclusions}

In conclusion, here we report for the first time the association of a common variant in the FTL1 gene with sepsis-induced ARDS susceptibility. Our findings demonstrate that the integration of information from different sources of public genomic data could be an efficient method to identify novel genes for ARDS susceptibility.

\section{Additional files}

Additional file 1: Table S1. Significant probe sets in the microarray-based differential gene expression analysis comparing experimental sepsis groups with a common control group (data obtained from Acosta-Herrera et al. PLoS One 2015, 10:e0132296). Description of data: statistics obtained from the pathway enrichment analysis performed for each experimental group included in the transcriptomic lung study before the prioritization of candidate genes. (XLS $431 \mathrm{~kb}$ )

Additional file 2: Table S2. Pathway enrichment analysis performed in each experimental septic group. Description of data: summary results of the pathway enrichment analysis. (DOC $51 \mathrm{~kb}$ )

Additional file 3: Table S3. Protein interaction network analysis (data obtained from Acosta-Herrera et al. PLoS One 2015, 10:e0132296). Summary results of the protein-protein interaction network analysis as part of the lung transcriptomic study previously published. (DOC $40 \mathrm{~kb}$ )

Additional file 4: Table S4. Summary results of the transcriptomic study in blood cells from septic and sepsis-derived ARDS patients. Summary results of the validation from GSEA in whole blood performed in a transcriptomic study. (DOC $31 \mathrm{~kb}$ )

Additional file 5: Table S5. Summary statistics of SNPs located at the pathways with SNPs significantly associated with ARDS by Christie et al. (PLoS One 2012, 7:e28268). Summary results of the prioritization performed from the 13 genes with significant SNPs at the GWAs of ARDS performed by Christie et al. (XLS $45 \mathrm{~kb}$ )

Additional file 6: Table 4. ARDS susceptibility association results in the discovery and replication studies. Summary of association results for the three SNPS associated with ARDS susceptibility in the discovery and replication studies. (DOC $40 \mathrm{~kb})$

\section{Abbreviations}

ARDS: Acute respiratory distress syndrome; BAL: Bronchoalveolar lavage; Cl: Confidence interval; dbGaP: Database of Genotypes and Phenotypes; DYNC2H1: Dynein cytoplasmic-2 heavy chain-1; ENCODE: Encyclopedia of DNA Elements; eQTL: Expression Quantitative Trait Loci; FC: Fold change; FDR: False discovery rate; FLT1: Fms-related tyrosine kinase 1; GWAS: Genome-wide association studies; HWE: Hardy-Weinberg equilibrium; ICU: Intensive care unit; ITGA1: Integrin, alpha-1; LD: Linkage disequilibrium; MAF: Minor allele frequency; MV: Mechanical ventilation; OR: Odds ratio; PEEP: Positive end-expiratory pressure; PPFIA1: Protein Tyrosine Phosphatase, Receptor Type F-interacting protein alpha 1; PRDX6: Peroxiredoxin-6; SIV: Septic animals ventilated with injurious mechanical ventilation; SNPs: Single nucleotide polymorphisms; SPV: Septic animals ventilated with protective mechanical ventilation; SS: Unventilated spontaneously breathing septic animals; STAT: Signal transducer and activator of transcription; VEGF: Vascular endothelial growth factor

\section{Acknowledgements}

Investigators of GENetics of SEPsis-induced ARDS (GEN-SEP) Network. Description of data: List of investigators that belong to the GEN-SEP Network.

Jesús Villar, Rosa L. Fernández (Hospital Universitario Dr. Negrín, Las Palmas de Gran Canaria); Carlos Flores, Maria Pino-Yanes, Marialbert Acosta-Herrera, Lina Pérez-Méndez, Almudena Corrales, Elena Espinosa, David Domínguez (Hospital Universitario NS de Candelaria, Santa Cruz de Tenerife); Jesús Blanco, Arturo Muriel (Hospital Universitario Río Hortega, Valladolid); Victor Sagredo, Juan C. Ballesteros (Hospital Clínico Universitario de Salamanca, Salamanca); Alfonso Ambrós, Rafael del Campo (Hospital General de Ciudad Real, Ciudad Real); Francisco Gandía, Leonor Nogales (Hospital Clínico de Valladolid); Rafael 
Fernández, Carles Subirá, (Fundació Althaia, Barcelona); Aurora Baluja (Hospital Clínico Universtario de Santiago de Compostela, La Coruña); José M. Añón, Elena González, Rosario Solano (Hospital Virgen de La Luz, Cuenca); Demetrio Carriedo, Francisco J. Diaz-Dominguez (Hospital General de León, León); Ramón Adalia, Elizabeth Zavala (Hospital Clínic, Barcelona).

\title{
Funding
}

This work was funded by Instituto de Salud Carlos III (CB06/06/1088, PI14/00844, Fl11/00074, PI16/00049, FI16/00136, PI17/00610), Agencia Canaria de Investigación, Innovación y Sociedad de la Información (TESIS2015010057) co-funded by European Social Fund, and by the European Regional Development Funds "A way of making Europe." MAH is recipient of a Juan de la Cierva fellowship (FJCl-2015-24028) from the Spanish Ministry of Economy, Industry and Competitiveness. The funders had no role in the study design, data collection and analysis, decision to publish, or preparation of the manuscript.

\section{Availability of data and materials}

The results of the lung transcriptomic study used to prioritize the candidate genes included in the discovery phase are publicly available on the ArrayExpress database (www.ebi.ac.uk/arrayexpress) under the accession number E-MTAB-2673. These were validated using data from a transcriptomic study in blood cells from septic and sepsis-derived ARDS patients (available at the NCBI GEO dataset under the accession number GSE10474) and the only GWAS of ARDS published at that time (results of the discovery phase available within the supporting information at https://doi.org/10.1371/ journal.pone.0028268.s003).

The replication dataset analyzed during the current study was obtained as part of the ARDSnet and the identification of SNPs Predisposing to Altered ALI Risk (iSPAAR) Consortium Genetic Study funded by the NHLBI (RC2 HL101779) and is available in the Genotypes and Phenotypes (dbGaP) repository (study accession number phs000631.v1.p1): https://www.ncbi.nlm.nih.gov/projects/gap/cgi-bin/study.cgi?study_id=phs000631.v1.p1

\section{Authors' contributions}

NHP, BGG, MPY, MAH, JV, and CF contributed in the analysis and interpretation of data. NHP, BGG, MPY, MAH, JV, and CF drafting the manuscript. AC, NHP, AA, LN, AM, EGH, FDD, EZ, JB, and SFM participated in the acquisition of data. JV and CF contributed in study design, acquisition of funding, and general supervision of research group. All authors participated in the critical revision and final approval of the manuscript.

\section{Ethics approval and consent to participate}

The study was performed according to The Code of Ethics of the World Medical Association (Declaration of Helsinki), and informed consent was obtained from all patients or from their appropriate surrogates. The Research Ethics Committees of all participant hospitals approved this study.

\section{Consent for publication}

Not applicable.

\section{Competing interests}

The authors declare that they have no competing interests.

\section{Publisher's Note}

Springer Nature remains neutral with regard to jurisdictional claims in published maps and institutional affiliations.

\begin{abstract}
Author details
${ }^{1}$ Research Unit, Hospital Universitario N.S. de Candelaria, Universidad de La Laguna, Santa Cruz de Tenerife, Spain. ${ }^{2}$ Research Unit, Hospital Universitario Dr. Negrin, Las Palmas de Gran Canaria, Spain. ${ }^{3} \mathrm{CIBER}$ de Enfermedades Respiratorias, Instituto de Salud Carlos III, Madrid, Spain. ${ }^{4}$ Genomics and Health Group, Department of Biochemistry, Microbiology, Cell Biology and Genetics, Universidad de La Laguna, La Laguna, Tenerife, Spain. ${ }^{5}$ Intensive Care Unit, Hospital General de Ciudad Real, Ciudad Real, Spain. IIntensive Care Unit, Hospital Clínico Universitario de Valladolid, Valladolid, Spain. ${ }^{7}$ Intensive Care Unit, Hospital Universitario Rio Hortega, Valladolid, Spain. ${ }^{8}$ Intensive Care Unit, Hospital Virgen de La Luz, Cuenca, Spain. ${ }^{9}$ Intensive Care Unit, Hospital General de León, León, Spain. ${ }^{10}$ Intensive Care Unit, Hospital Clinic Barcelona, Barcelona, Spain. "'Department of Anesthesiology, Hospital Clínico Universitario, Universidad de Valencia, Valencia, Spain. ${ }^{12}$ Division of Pulmonary and Critical Care Medicine, Department of Medicine, University of Chicago, Chicago, USA. ${ }^{13}$ Instituto de Parasitología y Biomedicina López-Neyra, IPBLN-CSIC, P.T.S, Granada, Spain.
\end{abstract}

Received: 10 January 2018 Accepted: 20 June 2018

Published online: 09 July 2018

\section{References}

1. Bernard GR, Artigas A, Brigham KL, Carlet J, Falke K, Hudson L, Lamy M, Legall JR, Morris A, Spragg R (1994) The American-European Consensus Conference on ARDS. Definitions, mechanisms, relevant outcomes, and clinical trial coordination. Am J Respir Crit Care Med 149:818-824. https://doi.org/10.1164/ajrccm.149.3.7509706

2. Villar J, Blanco J, Anon JM, Santos-Bouza A, Blanch L, Ambros A, Gandia F, Carriedo D, Mosteiro F, Basaldua S et al (2011) The ALIEN study: incidence and outcome of acute respiratory distress syndrome in the era of lung protective ventilation. Intensive Care Med 37:1932-1941. https://doi.org/10.1007/s00134-011-2380-4

3. Villar J, Blanco J, Kacmarek RM (2016) Current incidence and outcome of the acute respiratory distress syndrome. Curr Opin Crit Care 22:1-6. https://doi.org/10.1097/MCC.0000000000000266.

4. Rubenfeld GD, Caldwell E, Peabody E, Weaver J, Martin DP, Neff M, Stern EJ, Hudson LD (2005) Incidence and outcomes of acute lung injury. N Engl J Med 353:1685-1693. https://doi.org/10.1056/NEJMoa050333 
5. Matthay MA, Zimmerman GA (2005) Acute lung injury and the acute respiratory distress syndrome: four decades of inquiry into pathogenesis and rational management. Am J Respir Cell Mol Biol 33:319-327. https://doi.org/10.1165/rcmb.F305

6. Acosta-Herrera M, Pino-Yanes M, Perez-Mendez L, Villar J, Flores C (2014) Assessing the quality of studies supporting genetic susceptibility and outcomes of ARDS. Front Genet 5:20. https://doi.org/10.3389/fgene.2014.00020

7. Flores C, Pino-Yanes Mdel M, Villar J (2008) A quality assessment of genetic association studies supporting susceptibility and outcome in acute lung injury. Crit Care 12:R130. https://doi.org/10.1186/cc7098

8. Villar J, Perez-Mendez L, Blanco J, Anon JM, Blanch L, Belda J, Santos-Bouza A, Fernandez RL, Kacmarek RM (2013) A universal definition of ARDS: the $\mathrm{PaO} 2 / \mathrm{FiO}_{2}$ ratio under a standard ventilatory setting - a prospective, multicenter validation study. Intensive Care Med 39:583-592. https://doi.org/10.1007/s00134-012-2803-x

9. Martin GS, Mannino DM, Eaton S, Moss M (2003) The epidemiology of sepsis in the United States from 1979 through 2000. N Engl J Med 348:1546-1554. https://doi.org/10.1056/NEJMoa022139

10. Erickson SE, Shlipak MG, Martin GS, Wheeler AP, Ancukiewicz M, Matthay MA, Eisner MD (2009) Racial and ethnic disparities in mortality from acute lung injury. Crit Care Med 37:1-6

11. Linko R, Okkonen M, Pettila V, Perttila J, Parviainen I, Ruokonen E, Tenhunen J, Ala-Kokko T, Varpula T (2009) Acute respiratory failure in intensive care units. FINNALI: a prospective cohort study. Intensive Care Med 35:1352-1361

12. Christie JD, Wurfel MM, Feng R, O'Keefe GE, Bradfield J, Ware LB, Christiani DC, Calfee CS, Cohen MJ, Matthay M et al (2012) Genome wide association identifies PPFIA1 as a candidate gene for acute lung injury risk following major trauma. PLoS One 7:e28268. https://doi.org/10.1371/journal.pone.0028268

13. Acosta-Herrera M, Lorenzo-Diaz F, Pino-Yanes M, Corrales A, Valladares F, Klassert TE, Valladares B, Slevogt H, Ma SF, Villar J, Flores C (2015) Lung transcriptomics during protective ventilatory support in sepsis-induced acute lung injury. PLoS One 10:e0132296. https://doi.org/10.1371/journal.pone.0132296

14. Dennis G Jr, Sherman BT, Hosack DA, Yang J, Gao W, Lane HC (2003) Lempicki RA (2003) DAVID: database for annotation, visualization, and integrated discovery. Genome Biol 4:P3

15. Mura M, dos Santos CC, Stewart D, Liu M (2004) Vascular endothelial growth factor and related molecules in acute lung injury. J Appl Physiol 97:1605-1617. https://doi.org/10.1152/japplphysiol.00202.2004

16. Larrivee B, Freitas C, Suchting S, Brunet I, Eichmann A (2009) Guidance of vascular development: lessons from the nervous system. Circ Res 104:428-441. https://doi.org/10.1161/CIRCRESAHA.108.188144

17. Segura I, De Smet F, Hohensinner PJ, Ruiz de Almodovar C, Carmeliet P (2009) The neurovascular link in health and disease: an update. Trends Mol Med 15:439-451. https://doi.org/10.1016/j.molmed.2009.08.005

18. Howrylak JA, Dolinay T, Lucht L, Wang Z, Christiani DC, Sethi JM, Xing EP, Donahoe MP, Choi AM (2009) Discovery of the gene signature for acute lung injury in patients with sepsis. Physiol Genomics 37:133-139. https://doi.org/ 10.1152/physiolgenomics.90275.2008

19. The Gene Ontology Consortium (2017) Expansion of the Gene Ontology knowledgebase and resources. Nucleic Acids Res 45:D331-D338. https://doi.org/10.1093/nar/gkw1108

20. Kanehisa M, Goto S (2000) KEGG: kyoto encyclopedia of genes and genomes. Nucleic Acids Res 28:27-30. https:// doi.org/10.1093/nar/28.1.27

21. Cookson W, Liang L, Abecasis G, Moffatt M, Lathrop M (2009) Mapping complex disease traits with global gene expression. Nat Rev Genet 10:184-194. https://doi.org/10.1038/nrg2537

22. Nyholt DR (2004) A simple correction for multiple testing for single-nucleotide polymorphisms in linkage disequilibrium with each other. Am J Hum Genet 74:765-769. https://doi.org/10.1086/383251

23. Abecasis GR, Auton A, Brooks LD, DePristo MA, Durbin RM, Handsaker RE, Kang HM, Marth GT, McVean GA (2012) An integrated map of genetic variation from 1,092 human genomes. Nature 491:56-65. https://doi.org/10.1038/nature11632

24. Barrett JC, Fry B, Maller J, Daly MJ (2005) Haploview: analysis and visualization of LD and haplotype maps. Bioinformatics 21:263-265. https://doi.org/10.1093/bioinformatics/bth457

25. ARDS Definition Task Force, Ranieri VM, Rubenfeld GD, Thompson BT, Ferguson ND, Caldwell E, Fan E, Camporota L, Slutsky AS (2012) Acute respiratory distress syndrome: the Berlin definition. JAMA 307:2526-2533. https://doi. org/10.1001/jama.2012.5669

26. Spanish National DNA Biobank, Salamanca. http://www.bancoadn.org. Accessed 10 Sept 2014

27. R Development Core Team (2008) R: a language and environment for statistical computing, Viena, Austria. http:// www.R-project.org

28. Purcell S, Neale B, Todd-Brown K, Thomas L, Ferreira MA, Bender D, Maller J, Sklar P, de Bakker PI, Daly MJ, Sham PC (2007) PLINK: a tool set for whole-genome association and population-based linkage analyses. Am J Hum Genet 81:559-575. https://doi.org/10.1086/519795

29. Price AL, Patterson NJ, Plenge RM, Weinblatt ME, Shadick NA, Reich D (2006) Principal components analysis corrects for stratification in genome-wide association studies. Nat Genet 38:904-909. https://doi.org/10.1038/ng1847

30. Fuchsberger C, Abecasis GR, Hinds DA (2015) minimac2: faster genotype imputation. Bioinformatics 31:782-784. https://doi.org/10.1093/bioinformatics/btu704

31. McCarthy S, Das S, Kretzschmar W, Delaneau O, Wood AR, Teumer A, Kang HM, Fuchsberger C, Danecek P, Sharp $\mathrm{K}$ et al (2016) A reference panel of 64,976 haplotypes for genotype imputation. Nat Genet 48:1279-1283. https:// doi.org/10.1038/ng.3643

32. Das S, Forer L, Schonherr S, Sidore C, Locke AE, Kwong A, Vrieze SI, Chew EY, Levy S, McGue M et al (2016) Nextgeneration genotype imputation service and methods. Nat Genet 48:1284-1287. https://doi.org/10.1038/ng.3656

33. Han B, Eskin E (2011) Random-effects model aimed at discovering associations in meta-analysis of genome-wide association studies. Am J Hum Genet 88:586-598. https://doi.org/10.1016/j.ajhg.2011.04.014

34. Ward LD, Kellis M (2012) HaploReg: a resource for exploring chromatin states, conservation, and regulatory motif alterations within sets of genetically linked variants. Nucleic Acids Res 40:D930-D934. https://doi.org/10.1093/nar/gkr917

35. GTEx Consortium (2013) The genotype-tissue expression (GTEx) project. Nat Genet 45:580-585. https://doi.org/10. 1038/ng.2653

36. Rushefski M, Aplenc R, Meyer N, Li M, Feng R, Lanken PN, Gallop R, Bellamy S, Localio AR, Feinstein SI et al (2011) Novel variants in the PRDX6 gene and the risk of acute lung injury following major trauma. BMC Med Genet 12:77. https://doi.org/10.1186/1471-2350-12-77 
37. Matsumoto T, Mugishima H (2006) Signal transduction via vascular endothelial growth factor (VEGF) receptors and their roles in atherogenesis. J Atheroscler Thromb 13:130-135. https://doi.org/10.5551/jat.13.130

38. Hamada N, Kuwano K, Yamada M, Hagimoto N, Hiasa K, Egashira K, Nakashima N, Maeyama T, Yoshimi M, Nakanishi Y (2005) Anti-vascular endothelial growth factor gene therapy attenuates lung injury and fibrosis in mice. J Immunol 175:1224-1231. https://doi.org/10.4049/jimmunol.175.2.1224

39. Konta A, Ozaki K, Sakata Y, Takahashi A, Morizono T, Suna S, Onouchi Y, Tsunoda T, Kubo M, Komuro I et al (2016) A functional SNP in FLT1 increases risk of coronary artery disease in a Japanese population. J Hum Genet 61:435-441. https://doi.org/10.1038/jhg.2015.171

40. Maitre B, Boussat S, Jean D, Gouge M, Brochard L, Housset B, Adnot S, Delclaux C (2001) Vascular endothelial growth factor synthesis in the acute phase of experimental and clinical lung injury. Eur Respir J 18:100-106

41. Thickett DR, Armstrong L, Millar AB (2002) A role for vascular endothelial growth factor in acute and resolving lung injury. Am J Respir Crit Care Med 166:1332-1337. https://doi.org/10.1164/rccm.2105057

42. Abadie Y, Bregeon F, Papazian L, Lange F, Chailley-Heu B, Thomas P, Duvaldestin P, Adnot S, Maitre B, Delclaux C (2005) Decreased VEGF concentration in lung tissue and vascular injury during ARDS. Eur Respir J 25:139-146. https://doi.org/10.1183/09031936.04.00065504

43. Kaner RJ, Crystal RG (2001) Compartmentalization of vascular endothelial growth factor to the epithelial surface of the human lung. Mol Med 7:240-246

44. Ferrara N, Gerber HP, LeCouter J (2003) The biology of VEGF and its receptors. Nat Med 9:669-676. https://doi.org/ 10.1038/nm0603-669

45. Kaner RJ, Ladetto JV, Singh R, Fukuda N, Matthay MA, Crystal RG (2000) Lung overexpression of the vascular endothelial growth factor gene induces pulmonary edema. Am J Respir Cell Mol Biol 22:657-664. https://doi.org/ 10.1165/ajrcmb.22.6.3779

46. Deloukas P, Kanoni S, Willenborg C, Farrall M, Assimes TL, Thompson JR, Ingelsson E, Saleheen D, Erdmann J, Goldstein BA et al (2013) Large-scale association analysis identifies new risk loci for coronary artery disease. Nat Genet 45:25-33. https://doi.org/10.1038/ng.2480

47. Howson JMM, Zhao W, Barnes DR, Ho WK, Young R, Paul DS, Waite LL, Freitag DF, Fauman EB, Salfati EL et al (2017) Fifteen new risk loci for coronary artery disease highlight arterial-wall-specific mechanisms. Nat Genet 49: 1113-1119. https://doi.org/10.1038/ng.3874

48. McGinnis R, Steinthorsdottir V, Williams NO, Thorleifsson G, Shooter S, Hjartardottir S, Bumpstead S, Stefansdottir L, Hildyard L, Sigurdsson JK et al (2017) Variants in the fetal genome near FLT1 are associated with risk of preeclampsia. Nat Genet 49:1255-1260. https://doi.org/10.1038/ng.3895

49. Maynard SE, Min JY, Merchan J, Lim KH, Li J, Mondal S, Libermann TA, Morgan JP, Sellke FW, Stillman IE et al (2003) Excess placental soluble fms-like tyrosine kinase 1 (sFlt1) may contribute to endothelial dysfunction, hypertension, and proteinuria in preeclampsia. J Clin Invest 111:649-658. https://doi.org/10.1172/JCl17189

50. Lee JY, Lee BS, Shin DJ, Woo Park K, Shin YA, Joong Kim K, Heo L, Young Lee J, Kyoung Kim Y, Jin Kim Y et al (2013) A genome-wide association study of a coronary artery disease risk variant. J Hum Genet 58:120-126. https://doi.org/10.1038/jhg.2012.124

51. Shapiro NI, Schuetz P, Yano K, Sorasaki M, Parikh SM, Jones AE, Trzeciak S, Ngo L, Aird WC (2010) The association of endothelial cell signaling, severity of illness, and organ dysfunction in sepsis. Crit Care 14:R182. https://doi.org/ $10.1186 /$ cc9290

52. Skibsted S, Jones AE, Puskarich MA, Arnold R, Sherwin R, Trzeciak S, Schuetz P, Aird WC, Shapiro NI (2013) Biomarkers of endothelial cell activation in early sepsis. Shock 39:427-432. https://doi.org/10.1097/SHK. Ob013e3182903f0d

53. Perkins GD, Roberts J, McAuley DF, Armstrong L, Millar A, Gao F, Thickett DR (2005) Regulation of vascular endothelial growth factor bioactivity in patients with acute lung injury. Thorax 60:153-158. https://doi.org/10. 1136/thx.2004.027912

54. Kendall RL, Thomas KA (1993) Inhibition of vascular endothelial cell growth factor activity by an endogenously encoded soluble receptor. Proc Natl Acad Sci U S A 90:10705-10709

55. Thomas D (2006) Statistical methods in genetic epidemiology. Int J Epidemiol. USA, Oxford University Press; p. 501

56. Neupane B, Walter SD, Krueger P, Loeb M (2010) Community controls were preferred to hospital controls in a case-control study where the cases are derived from the hospital. J Clin Epidemiol 63:926-931. https://doi.org/10. 1016/j.jclinepi.2009.11.006

57. Weiss ST (2001) Association studies in asthma genetics. Am J Respir Crit Care Med 164:2014-2015. https://doi.org/ 10.1164/ajrccm.164.11.2110043b 